\section{P16 GENDER DIFFERENCES IN WALKING (FOR LEISURE, TRANSPORT AND IN TOTAL) ACROSS ADULT LIFE: A SYSTEMATIC REVIEW}

TM Pollard*, JM Wagnild. Department of Anthropology, Durham University, Durham, UK

10.1136/jech-2017-SSMAbstracts. 118

Background The possibility of gender differences in walking practices has largely been ignored, but an understanding of whether and how walking practices differ between men and women would usefully inform interventions to promote walking. The aim of this systematic review was to examine gender differences in walking for leisure, transport and in total in adults, and to assess whether gender differences in walking practices change across the adult life-course.

Methods Web of Science Core Collections, PubMed and the Transportation Research International Documentation databases were searched to identify relevant studies, as well as reference lists of included studies. Papers providing quantitative data on the walking of both men and women aged 18 years and above in a high-income country and published between 1995 and 2015 were identified. Data assessed as high quality were extracted and results were synthesised using forest plots and narrative summary.

Results Of the 24498 studies retrieved by the search strategy, 36 studies were included. Most (30) studies reported data on the prevalence of walking for a minimum duration, usually $10 \mathrm{~min}$ per week, either in total or for a specific purpose. Of these, 6 of 6 studies combining all ages found that more women than men walked for leisure (Odds Ratios (ORs) 1.12-1.47). There was no evidence of a gender difference in overall walking for transport (ORs $0.78-1.11$ for 3 studies combining all ages), but 3 of 3 studies found that more women than men walked to undertake errands (ORs 1.082.97). 5 of 6 studies combining all ages reported no gender difference in total walking (ORs 0.90-1.39). 6 studies reported on walking for leisure by age group; more young women than young men walked for leisure (ORs 1.54-1.99), but this difference consistently declined with age such that in the oldest age groups it had disappeared or reversed (ORs 0.63-0.96). There was some evidence for a similar pattern in overall walking.

Conclusion While we found no gender difference in participation in walking overall, we did find consistent gender differences in participation in walking for some purposes, including for leisure, and that there are gender differences in the impact of age on walking. We conclude that more research is needed to improve our understanding of how walking fits into the lives of women and men across the life-course, especially in relation to gender differences in the impact of ageing on walking.

\section{P17 CHRONIC MALNUTRITION IN EARLY LIFE AND COGNITIVE OUTCOME IN MID-CHILDHOOD: EVIDENCE FROM FOUR DEVELOPING COUNTRIES}

Z ZHANG*, T CHANDOLA. CMIST, University of Manchester, Manchester, the UK

10.1136/jech-2017-SSMAbstracts.119

Background Chronic malnutrition is a major global health challenge, particularly in low- and middle-income countries (LMICs). Malnutrition, in particularly stunting in early childhood, is associated with developmental delay, including cognitive deficits and poor academic performance, often leading to reduced work capacity in adulthood. Few studies have examined the long-term influence of chronic malnutrition in early life on subsequent cognitive outcome and how recovering from early stunting can benefit cognitive development in a cross-national context.

Methods Analysing data on 8062 children in Ethiopia $(n=1,999)$, India $(2,011)$, Peru $(n=2,052)$ and Vietnam $(n=2,000)$ from the Young Lives Study, a cross-national cohort study on child poverty and wellbeing in the developing world, we studied younger cohort, enrolled in 2002 at ages 6-17.9 months (round 1), followed in 2006 at 5 years old (round 2) and in 2009 at 8 years old (round 3) to explore whether duration of chronic malnutrition in early life matters for cognitive outcome and whether recovery from early stunting benefits for cognitive development.

The duration of stunting was defined as having a HAZ Height-for-Age $Z$ scores (HAZ) $<-2$ based on WHO standards. Chronic malnutrition was determined as the number of stunting for three rounds, ranging from 0 to 3 . Recovery from stunting was defined as having a HAZ >-2 in subsequent rounds after baseline. Cognitive outcome was assessed at age 8 using the Peabody Picture Vocabulary Test (PPVT) to evaluate vocabulary skills and listening comprehension.

Results Longer term of being stunted was associated with lower cognitive scores at age 8 for both girls $(p<0.05)$ and boys $(p<0.01)$. We found catch-up growth from early stunting significantly increases cognitive outcomes of girls who were stunted at baseline (coef=7.91, p<0.01). No significant associations were found for boys. Chronic malnutrition during childhood can be detrimental for children's cognitive development in later life. Recovery from early stunting is possible, in particular for girls and this can lead to improvement in cognitive development.

Conclusion Policy makers and program planners should consider redoubling efforts to prevent stunting and promote catch-up growth over the first few years of life, especially for girls, as a way of improving children's physical and intellectual development. Given the important role girls and women play in contributing to national and global economies, improving nutrition in early childhood of girls in developing countries is a long-term economic investment and driver of economic growth.

\section{P18 IS THE RISK OF PERMANENT CHILDHOOD HEARING IMPAIRMENT HIGHER IN BABIES OF SHORTER GESTATIONAL LENGTH?}

E Butcher*, C Dezateux, R Knowles. Life Course Epidemiology and Biostatistics, Great Ormond Street Institute of Child Health, UCL, London, UK

10.1136/jech-2017-SSMAbstracts. 120

Background Permanent childhood hearing impairment (PCHI) has major implications for social and cognitive development over the life-course. Early detection and management improves outcomes, providing the rationale for universal newborn hearing screening (UNHS), introduced in England from 2002. In England and Wales, one baby in every eight is admitted to specialist neonatal care, including neonatal intensive or special care baby units (NICU/SCBU), a known risk factor for PCHI, however the association with gestational length remains 
unclear. In England and Wales, 7.6\% infants are born before 37 weeks gestation; this is higher (16.2\%) among those of South Asian ethnicity. We examined PCHI risk by age 11 years in a prospective UK-wide cohort of children born before UNHS. We tested the hypothesis that lower gestational length is associated with higher PCHI risk after adjusting for NICU/ SCBU admission and ethnicity.

Methods PCHI risk (cumulative incidence) was based on parental report of hearing impairment and associated treatment at ages nine months, three, five, seven and 11 years for 19518 children participating in the Millennium Cohort Study. The association of PCHI (defined by provision of hearing aids/cochlear implants, persisting hearing impairment at final report, and absence of glue ear) with gestational length was investigated using multivariable discrete-time survival analysis, adjusting for NICU/SCBU admission, ethnicity and other confounding factors, and weighting for survey design (Stata: Release 14; StataCorp LP).

Results Parents reported no health problems in the first week after birth in 10247 (52.4\%), children, neonatal illness without NICU/SCBU admission in 6781 (38.0\%), and NICU/SCBU admission in 1785 (9.6\%). PCHI was ascertained in 44 children by age 11 years. PCHI risk was 1.0 per 1000 children (95\% CI: $0.6-1.6)$ by age 9 months, rising by age five to 1.5 $(1.0-2.2)$ and by age 11 to $2.1(1.5-3.0)$. PCHI risk by age 11 was not associated with gestational length (hazard ratio (HR): 1.00, 95\% CI: 0.98-1.03), but was associated with parental report of neonatal illness with or without NICU/ SCBU admission (HR: 6.33; 2.27-17.63 and 2.62; 1.15-5.97, respectively) and Bangladeshi or Pakistani ethnicity (HR: 2.78; 1.06-7.31).

Conclusion In this cohort, born before UNHS, PCHI risk was highest in infancy. Neonatal illness, irrespective of NICU/ SCBU admission, and not gestational length, increased PCHI risk by age 11 years. Further research should explore the observed increased PCHI risk in children of Bangladeshi or Pakistani ethnicity, and the relevance for UNHS of PCHI with onset or diagnosis after infancy.

ESRC-funded PhD ES/J500185/1.

\section{\begin{tabular}{|l|l}
\hline P19 & FATHER INVOLVEMENT AND ATTITUDES IN EARLY
\end{tabular} CHILD-REARING AND DEPRESSIVE SYMPTOMS IN THE PRE-ADOLESCENT PERIOD IN A UK BIRTH COHORT}

C Opondo*, M Redshaw, M Quigley. Nuffield Department of Population Health, University of Oxford, Oxford, UK

\subsection{6/jech-2017-SSMAbstracts. 121}

Background Although much of the research on the influence of parenting on child development has emphasised the mother's role, increasing evidence highlights the important role of fathers in the development, health and well-being of their children. In this study we aimed to investigate the association between father involvement in early child upbringing and depressive symptoms in the same children in their pre-teenage years.

Methods Data for the study came from the Avon Longitudinal Study of Parents and Children (ALSPAC) cohort from the southwest of England. The outcome, depressive symptoms, was measured using the short Moods and Feelings Questionnaire (sMFQ) when the children were 9 and 11 years of age. The primary exposure was paternal involvement measured using scores derived from factor analysis of fathers' report of their participation in, understanding of, and feelings about their child's early upbringing.

Results Three factors were identified in the factor analysis. Scores on factor 1 measured fathers' emotional response to the child; scores on factor 2 measured the frequency of fathers' involvement in domestic and childcare activities; scores on factor 3 measured fathers' feelings of security in their role as parent and partner. Children of fathers with high scores on factors 1 and 3 had 12\% (OR 0.88, 95\% CI 0.81-0.97, $\mathrm{p}=0.009$ ) and $9 \%$ (OR 0.91, 95\% CI 0.82-1.00, $\mathrm{p}=0.040$ ) respectively lower adjusted odds of reporting more depressive symptoms at 9 years. There was no evidence of a difference in depressive symptoms associated with factor 1 and factor 3 scores at 11 years. However, there was weak evidence of a $13 \%$ increase in odds of reporting more depressive symptoms associated with 1 unit increase in factor 2 scores at age 9 years (OR 1.13, 95\% CI 0.99-1.29, p=0.061) and a $16 \%$ increase at 11 years (OR 1.16, 95\% CI 1.01-1.34, p=0.040). Conclusion Positive psychological and emotional aspects of father involvement in children's early upbringing, but not the quantity of direct involvement in childcare, may protect children against developing symptoms of depression in their preteen years.

\section{\begin{tabular}{|l|l}
\hline P20 CUMULATIVE EFFECT OF ADVERSE CHILDHOOD \\
\hline
\end{tabular} EXPERIENCES ON AFFECTIVE SYMPTOM TRAJECTORIES IN ADULTHOOD: EVIDENCE FROM A BRITISH BIRTH COHORT}

${ }^{1}$ EJ Thompson*, ${ }^{2} \mathrm{M}$ Richards, ${ }^{1} \mathrm{D}$ Gaysina. 'School of Psychology, University of Sussex, Brighton, UK; ${ }^{2}$ MRC Unit for Lifelong Health and Ageing, University College London, London, UK

\subsection{6/jech-2017-SSMAbstracts. 122}

Background Previous studies have shown that specific types of adverse childhood events (ACEs), such as parental divorce and parental psychopathology, pose a risk for the development of affective symptoms in adulthood (AS). However, a majority of this evidence is based on single types of retrospectively reported ACEs. This is problematic as ACEs tend to be interrelated and often co-occur.

Methods We used the data from the MRC National Survey of Health and Development (NSHD). This is an ongoing longitudinal study of 5362 women and men who were born in Britain in1946. Later life AS were measures using the General Health Questionnaire (GHQ) at ages 53 y, 60-64 y and 69.

Multiple imputation was implemented on each ACE predictor and a cumulative risk index was derived though summing the number of adversities experienced by each participant $(0$, $1,2,3 \ldots 20)$ before age $16 \mathrm{y}$. The effect of cumulative ACEs on AS at each time point (53, 60-64 and 69) was examined using linear regression.

Results Preliminary analyses revealed a significant association was found between cumulative ACEs and AS at ages 60-64, $\beta(1,2183)=0.07, p=0.002$, and $69, \quad \beta(1,2110)=0.07$, $\mathrm{p}=0.003$, but not age $53 \beta(1,2900)=0.04, \mathrm{p}=0.058$. Further to this growth mixture modelling will be used to model latent trajectories of AS between age 53 and 69 years and the effect of cumulative ACEs will be examined.

Discussion These findings will be presented in light of the growing evidence for the negative effects of ACEs on health and wellbeing in later life. Furthermore, we will discuss how 